Original research article

\title{
Antepartum and postpartum changes in adipokines, endothelial dysfunction, inflammatory markers and other biochemical parameters in preeclamptic women: A prospective observational cohort study
}

\author{
Amany Yasseen Talab ${ }^{1}$, Haitham Aboali Hamza ${ }^{2}$, Tarek Mohamed Mostafa ${ }^{1}$ \\ ${ }^{1}$ Tanta University, Faculty of Pharmacy, Department of Clinical Pharmacy, Tanta, Egypt \\ ${ }^{2}$ Menofya University, Faculty of Medicine, Department of Obstetrics \& Gynecology, Shibin El Kom, Egypt
}

\begin{abstract}
This study aimed at evaluating the role played by insulin resistance, lipid metabolism disorder, oxidative stress, resistin, vaspin, Interleukin-18 and asymmetric dimethyl arginine as a marker for endothelial dysfunction in the pathogenesis of preeclampsia.

This prospective observational cohort study involved 60 women who were classified into: 20 non-pregnant women (group 1 or control group), 20 normally pregnant women (group 2) and 20 preeclamptic women (group 3) at their third trimester.

The pregnant women were assessed at their third trimester and further re-evaluated four weeks after delivery. The assessment included demography, assessment of proteinuria and urinary protein to creatinine ratio, blood pressure measurement and assessment of fasting blood glucose, fasting insulin level, lipid panel and the circulating levels of malondialdehyde, resistin, vaspin, interleukin-18 and asymmetric dimethyl arginine.

Preeclamptic women showed more atherogenic lipid profile, significantly higher Homeostatic Model Assessment for Insulin Resistance (HOMA-IR) and significantly elevated levels of malondialdehyde, resistin, vaspin and interleukin-18 than the other study groups. Serum asymmetric dimethyl arginine concentration showed non-significant difference among the three study groups. The levels of resistin and vaspin showed significant decrease four weeks postpartum in preeclamptic group.

We concluded that, preeclampsia was associated with insulin resistance, dyslipidemia, oxidative stress, inflammation and significant changes in adipokines; resistin and vaspin. Furthermore, the significant increase in the serum levels of resistin and vaspin at the third trimester and their significant decline four weeks postpartum in preeclamptic group focus the attention on the role played by these adipokines in the pathogenesis of preeclampsia.
\end{abstract}

Keywords: Asymmetric dimethyl arginine; Interleukin-18; Malondialdehyde; Preeclampsia; Resistin; Vaspin

\section{Highlights:}

- Preeclampsia was associated with insulin resistance; dyslipidemia, lipid peroxidation and inflammation giving suggestion about the role played by insulin resistance, dyslipidemia, lipid peroxidation and inflammation in the pathogenesis of preeclampsia.

- Significant increase in the serum resistin and vaspin levels at third trimester which tended to decline 4 weeks postpartum was observed in preeclamptic women which focus the attention on the role played by these adipokines during preeclampsia.

\section{Introduction}

Preeclampsia is a multi-systemic progressive disorder that occurs after 20 weeks of gestation and may last for 4-6 weeks postpartum. International Society for the Study of Hypertension in Pregnancy (ISSHP) defined preeclampsia as hypertension (blood pressure 140/90 $\mathrm{mmHg}$ ) coexists with one or more of the following new-onset conditions; proteinuria and maternal organ dysfunction including neurological complications, renal impairment, hematological complications and uteropla- cental dysfunction (Tranquilli et al., 2014). Preeclampsia has a negative impact on both mother and fetus and may be associated with maternal mortality (Ghulmiyyah and Sibai, 2012).

The pathogenesis of preeclampsia is related to insulin resistance, lipid metabolism disorder and endothelial dysfunction (Abhari et al., 2014). Oxidative stress is considered as a leading cause for placental vasoconstriction and reduction of uteroplacental circulation that in turn stimulate pro-inflammatory cytokines and anti-angiogenic factors release which participate in endothelial dysfunction and placental hypoxia (de Lucca et al., 2016).

\footnotetext{
* Corresponding author: Amany Yasseen Talab, Tanta University, Faculty of Pharmacy, Department of Clinical Pharmacy, Tanta, Egypt 31111; e-mail: amany_yasseen@yahoo.com http://doi.org/10.32725/jab.2021.004

Submitted: 2020-08-30 • Accepted: 2021-01-22 • Prepublished online: 2021-01-28

J Appl Biomed 19/1: 62-72 • EISSN 1214-0287 • ISSN 1214-021X

(c) 2021 The Authors. Published by University of South Bohemia in České Budějovice, Faculty of Health and Social Sciences.

This is an open access article under the CC BY-NC-ND license.
} 
Asymmetric dimethyl arginine (ADMA) is considered as a mediator of endothelial dysfunction and vascular malfunctions. Therefore, ADMA may serve as a potential biomarker of endothelial dysfunction during preeclampsia (Tousoulis et al., 2015). Resistin and vaspin are adipokines secreted by adipose tissue. Resistin impairs glucose uptake by adipocytes, promotes inflammation and insulin resistance (Steppan et al., 2001). Vaspin is an adipokine which enhances insulin sensitivity and plays a role in glucolipid metabolism (Roca-Rodríguez et al., 2012).

The role which resistin and vaspin play in preeclampsia is still obscure. However, resistin induces endothelial cells activation by promoting endothelin-1 (Verma et al., 2003). Furthermore, resistin increases the expressions of various proinflammatory factors and inhibits endothelial nitric oxide synthase via oxidative stress in human endothelial cells (Chen et al., 2010; Hsu et al., 2011). Proinflammatory cytokines and oxidative stress are involved in the development of the endothelial dysfunction which results in abnormal placentation (Roberts, 1998; Shah and Khalil, 2015). Abnormal placentation, the starting point for the development of preeclampsia leads to placental ischemia and hypoxia with subsequent raised secretion of antiangiogenic factors including soluble fms-like tyrosine kinase-1 (sFlt-1) which antagonize the proangiogenic factors like placental growth factor (PlGF) (Lockwood et al., 2008). This may impair the antiangiogenic proangiogenic balance with subsequent increase in sFlt-1/ PlGF ratio which represents a reliable tool for early prediction and monitoring of preeclampsia (Ohkuchi et al., 2010). On the other hand, vaspin was reported to play an important role in prevention of endothelial injuries, suppression of inflammation and inhibition of reactive oxygen species (Hida et al., 2005; Phalitakul et al., 2011). Therefore, vaspin may provide a protective effect during preeclampsia.

In this context, the present study aimed at examining the metabolic and biochemical changes involved in the pathogenesis of preeclampsia including insulin resistance, lipid metabolism disorder, oxidative stress, adipokines (vaspin and resistin), inflammatory cytokine IL-18 and asymmetric dimethyl arginine (ADMA); a potential biomarker for endothelial dysfunction.

\section{Materials and methods}

\section{Study design}

This prospective observational cohort study was conducted on a total number of 60 women who were matched for age and BMI and ethnicity. All enrolled women were Egyptians which refers to the ethnic group and the nationality. The women were classified into: 20 apparently healthy non-pregnant women (group 1 or control group; $n=20$ ), 20 normally pregnant women (group 2; $n=20$ ) and 20 pregnant women with preeclampsia (group $3 ; n=20$ ) at their third trimester. Preeclampsia was defined and diagnosed by the elevation of blood pressure with systolic BP $\geq 140 \mathrm{~mm} \mathrm{Hg}$ and/or diastolic BP $\geq 90 \mathrm{~mm} \mathrm{Hg}$ in two separate occasions after 20 weeks of gestation in women with previously normal blood pressure. Preeclampsia was also defined by urinary protein to creatinine ratio greater than $0.3 \mathrm{mg} / \mathrm{mg}$. All participants were selected from the Outpatient Clinic of Obstetrics and Gynecology Department at the Menofya University Hospital including the healthy non-pregnant women who were in attendance for their routine medical check-up. The study was performed in accordance with the ethical standards as laid down in the 1964 declaration of
Helsinki and its later amendments. The institutional Research Ethics Committee approved the current study (CP0002). The study was registered as a clinical trial with ID: NCT04455204. Written informed consents were taken from all participants. The gestational age was determined conventionally and re-affirmed by ultrasonographic measurement. At enrollment, all participants were assessed and submitted to blood and urine samples collection. The pregnant women were further re-evaluated four weeks after delivery. Exclusion criteria were women with risk factors for oxidative stress such as smokers and those with medical history of diabetes mellitus and tuberculosis. Women with medical history of hypertension, familial hyperlipidemia, liver dysfunction, inflammatory diseases and renal disorder were excluded. Non-pregnant women on hormonal therapy or hormonal contraceptives were also excluded.

\section{Methods}

Demographic and anthropometric measurements

A detailed history including age and pregnancy history (parity, gravidity) was taken and the gestational age was determined. Height and weight were measured and body mass index (BMI) was calculated as follow: BMI $=\left[\right.$ Weight $\left.(\mathrm{kg}) \div \operatorname{Height}^{2}(\mathrm{~m})\right]$.

\section{Laboratory analysis}

Routine measurements

At enrollment, urine analysis was done on 10-20 ml of freshly voided mid-stream early morning urine samples and proteinuria was immediately assessed using urine dipstick test. Spot mid-stream urine specimens were also used for the determination of Protein: Creatinine Ratio (P/C ratio). Total urine protein concentration was evaluated through pyrogallol-red molybdate colorimetric method (Spectrum Diagnostic, Egypt) and the urinary creatinine concentration was measured by Jaffe colorimetric kinetic method (Spectrum Diagnostic, Egypt). The spot urine $\mathrm{P} / \mathrm{C}$ ratio was calculated by dividing the urinary protein concentration by the urinary creatinine concentration. Proteinuria was defined as +1 protein value with urine dipstick or a spot urine $\mathrm{P} / \mathrm{C}$ ratio cut off point of $(0.3 \mathrm{mg} / \mathrm{mg})$ (Brown et al., 2001). For each participant, measurement of blood pressure was done in two separate occasions using sphygmomanometer.

\section{Biochemical measurements}

At early morning, venous blood was collected from all participants after an overnight fasting. Blood was allowed to clot, centrifuged at $4500 \times \mathrm{g}$ for $10 \mathrm{~min}$ (Hettich Zentrifugen EBA 20). The serum was divided into two portions; the first portion (fresh sera) was used for immediate determination of fasting blood glucose and lipid profile and the second portion was frozen at $-80{ }^{\circ} \mathrm{C}$ until biochemical analysis of the remaining parameters. Both total cholesterol (TC) and triglyceride (TG) levels were assayed by enzymatic colorimetric method. High density lipoprotein (HDL-C) was assayed by precipitation method. Low-density lipoprotein cholesterol (LDL-C) and very low density lipoprotein (VLDL) were calculated using the Friedewald formula: [LDL-C = TC $-\mathrm{HDL}-\mathrm{C}-(\mathrm{TG} \div 5)$ ] and $[\mathrm{VLDL}=(\mathrm{TG} \div 5)]$ provided that TG level is less than $400 \mathrm{mg} /$ dl (Friedewald et al., 1972). Castelli risk index-I (TC/HDL-C) and Castelli risk index-II (LDL/HDL-C) were also calculated. Fasting blood glucose was assayed by glucose oxidase method (Spinreact, Spain). Fasting insulin level was assayed by ELISA kit (DRG International, Inc.). Estimation of insulin resistance (IR) was done using the HOMA-IR index which is defined as fasting insulin $(\mu \mathrm{IU} / \mathrm{ml})$ times fasting glucose $(\mathrm{mmol} / \mathrm{l})$ divided by 22.5 or divided by 405 if fasting blood glucose was 
expressed in mass units (mg/dl) (Matthews et al., 1985). Malondialdehyde (MDA) level was measured spectrophotometrically using Draper and Hadly method (Draper and Hadley 1990). Serum resistin, vaspin, IL-18 and ADMA concentrations were assayed using commercially available ELISA kits (BioVendor, Immunodignostic AG, eBioscience, Elbascience) according to the manufacturer's instructions.

\section{Statistical analysis}

The collected data were statistically analyzed using SPSS software (Statistical Package for the Social Sciences, version 19, SPSS Inc. Chicago, USA). Quantitative data were described as range, mean, standard deviation and median. Qualitative data were described as number and percent and comparison between groups was done using Chi-square test $\left(\chi^{2}\right)$. For comparison between means of two groups of parametric data of independent samples, student $t$-test was used. For comparison between means of two groups of non-parametric data of independent samples, $Z$ value of Mann-Whitney test was used. For comparison between means of two related groups (before and after data) of parametric data, paired $t$-test was used. For comparison between more than two means of parametric data, $F$ value of ANOVA test was calculated and scheffe test was used to compare between each two means if $F$ value was significant. Significant level was set at $p<0.05$.

\section{Results}

Tables 1 and 2 illustrate the basic and reproductive data of the study participants. At enrollment, ages and BMI of the women in the three study groups were statistically similar $(p>0.05)$. Non-significant difference in both systolic and diastolic blood pressure was observed between non-pregnant women and women with normal pregnancy $(p>0.05)$. However, both systolic and diastolic blood pressure were significantly elevated in preeclamptic women as compared to the other two groups
( $p<0.0001$ ). At enrollment, the results obtained from the dipstick test showed absence of proteinuria in both non-pregnant and normally pregnant women. On the other hand, four preeclamptic women $(20 \%)$ had +1 , ten women $(50 \%)$ had +2 , four women (20\%) had +3 and two women (10\%) had +4 . Non-significant difference was observed in urinary $\mathrm{P} / \mathrm{C}$ ratio between non-pregnant and normally pregnant women $(p=0.985)$. Women with preeclampsia showed significantly higher $\mathrm{P} / \mathrm{C}$ ratio as compared to non-pregnant and normally pregnant women $(p=0.000)$. At enrollment, there was non-significant difference between the normally pregnant women and those with preeclampsia regarding gestational age $(p=0.69)$, mean gravidity $(p=0.056)$ and mean parity $(p=0.95)$. The gestational age at delivery time was significantly lower in women with preeclampsia as compared to normally pregnant women $(p=0.0001)$. The neonates of the women with preeclampsia showed significantly lower birth weight than the women with normal pregnancy $(p=0.0001)$. Regarding mode of delivery, the number and percent of women who underwent cesarean section were significantly higher in women with preeclampsia as compared to those with normal pregnancy $(p=0.011)$. The time gap between enrollment and delivery time was $3.57 \pm 1.45$ weeks for normally pregnant women versus $2.35 \pm 0.87$ weeks for preeclamptic women $(p=0.009)$.

Tables 3 and 4 with Fig. 1 and 2 summarize the biochemical data of the study groups at enrollment. Fasting blood glucose, fasting insulin level and HOMA-IR index were significantly elevated in preeclamptic group than normally pregnant and non-pregnant groups $\left(P_{2} \& P_{3}=0.0001\right)$. TC, TG, LDL-C and VLDL were significantly elevated in preeclamptic women than the other two groups. Also, normally pregnant women showed significantly higher levels of TC, TG, VLDL and HDL-C than non-pregnant group. Mean HDL-C level was significantly higher in normally pregnant women than preeclamptic women $\left(P_{3}=0.0001\right)$. Castelli risk indices I and II were significantly higher in preeclamptic women than normally pregnant and non-pregnant women $\left(P_{2} \& P_{3}=0.0001\right)$.

Table 1. Basic data of the three study groups $(n=60)$

\begin{tabular}{|c|c|c|c|c|c|}
\hline Parameter & $\begin{array}{l}\text { Non-pregnant women } \\
(\mathrm{G} 1 ; n=20)\end{array}$ & $\begin{array}{l}\text { Normally pregnant women } \\
(G 2 ; n=20)\end{array}$ & $\begin{array}{l}\text { Pregnant women with } \\
\text { preeclampsia }(G 3 ; n=20)\end{array}$ & F value & $P$ \\
\hline \multicolumn{6}{|l|}{ Age (years) } \\
\hline Range & $23.00-38.00$ & $19.00-38.00$ & $20.00-45.00$ & \multirow{3}{*}{3.123} & \multirow{3}{*}{0.052} \\
\hline Mean \pm SD & $29.75 \pm 4.69$ & $26.25 \pm 5.27$ & $30.50 \pm 7.01$ & & \\
\hline Median & 29.00 & 25.00 & 30.00 & & \\
\hline \multicolumn{6}{|l|}{ BMI $\left(\mathrm{kg} / \mathrm{m}^{2}\right)$} \\
\hline Range & $22.70-35.20$ & $25.80-35.50$ & $25.30-41.60$ & \multirow{3}{*}{2.901} & \multirow{3}{*}{0.063} \\
\hline Mean \pm SD & $28.66 \pm 3.21$ & $30.18 \pm 2.54$ & $31.37 \pm 4.61$ & & \\
\hline Median & 27.10 & 29.70 & 30.25 & & \\
\hline \multicolumn{6}{|l|}{ SBP (mmHg) } \\
\hline Range & 100-120 & 90-120 & 140-190 & \multirow{3}{*}{98.7} & \multirow{3}{*}{$<0.0001^{*}$} \\
\hline Mean \pm SD & $114.50 \pm 6.86$ & $112.00 \pm 8.94$ & $156.50 \pm 15.90$ & & \\
\hline Median & 120 & 110 & 150 & & \\
\hline \multicolumn{6}{|l|}{$\mathrm{DBP}(\mathrm{mmHg})$} \\
\hline Range & 70-85 & 60-80 & 90-110 & \multirow{3}{*}{89.15} & \multirow{3}{*}{$<0.0001^{*}$} \\
\hline Mean \pm SD & $76.25 \pm 5.10$ & $74.75 \pm 6.78$ & $98.75 \pm 7.05$ & & \\
\hline Median & 80 & 80 & 100 & & \\
\hline \multicolumn{6}{|c|}{ U. $\mathrm{P} / \mathrm{C}$ ratio $(\mathrm{mg} / \mathrm{mg})$} \\
\hline Range & $0.12-0.47$ & $0.13-0.49$ & $0.36-1.10$ & \multirow[t]{2}{*}{59.836} & \multirow[t]{2}{*}{$0.000^{*}$} \\
\hline Mean \pm SD & $0.222 \pm 0.083$ & $0.23 \pm 0.089$ & $0.68 \pm 0.233$ & & \\
\hline
\end{tabular}

Data are presented as range, mean \pm SD and median. BMI: body mass index; SBP: Systolic blood pressure; DBP: diastolic blood Pressure; U. P/C ratio: Urinary protein to creatinine ratio. Significance level was set at $p<0.05$. ${ }^{*}$ Significant difference. 
Table 2. Reproductive data of the study participants

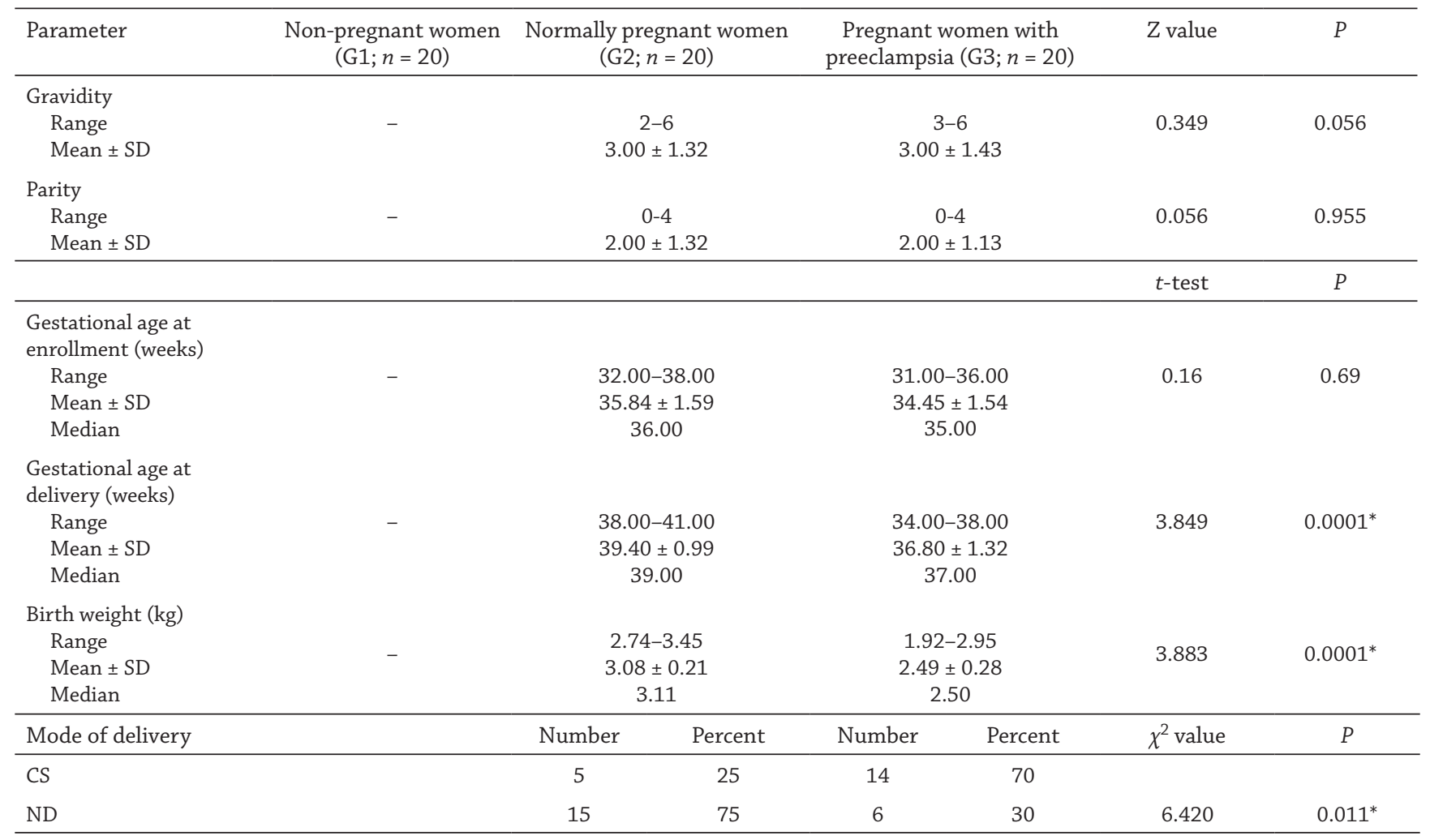

Data are presented as range, mean $\pm S D$, median, number and percent. $C$ s: cesarean section; ND: normal delivery; $Z$ value: $Z$ value of Mann-Whitney test, $\chi^{2}$ : Chi Square test. ${ }^{*}$ Significant difference $(p<0.05)$.

Table 3. Glycemic and lipid panels among the three study groups at enrollment $(n=60)$

\begin{tabular}{|c|c|c|c|c|c|}
\hline $\begin{array}{l}\text { Laboratory } \\
\text { findings }\end{array}$ & $\begin{array}{l}\text { Non-pregnant women } \\
(G 1 ; n=20)\end{array}$ & $\begin{array}{l}\text { Normally pregnant } \\
\text { women }(G 2 ; n=20)\end{array}$ & $\begin{array}{c}\text { Pregnant women with } \\
\text { preeclampsia }(G 3 ; n=20)\end{array}$ & $\begin{array}{l}\text { F value } \\
\quad P\end{array}$ & Scheffe test $(P)$ \\
\hline FPG (mg/dl) & $\begin{array}{l}51.00-96.00 \\
69.89 \pm 11.07\end{array}$ & $\begin{array}{l}70.00-105.00 \\
88.50 \pm 11.51\end{array}$ & $\begin{array}{c}74.00-144.00 \\
112.80 \pm 15.94\end{array}$ & $\begin{array}{l}54.553 \\
0.000^{*}\end{array}$ & $\begin{array}{c}P_{1} \& P_{2}=0.0001^{*} \\
P_{3}=0.0001^{*}\end{array}$ \\
\hline FPI $(\mu \mathrm{IU} / \mathrm{ml})$ & $\begin{array}{c}1.10-8.40 \\
4.60 \pm 1.95\end{array}$ & $\begin{array}{c}5.60-17.10 \\
10.22 \pm 3.75\end{array}$ & $\begin{array}{c}8.40-34.10 \\
20.18 \pm 6.89\end{array}$ & $\begin{array}{l}57.115 \\
0.0001^{*}\end{array}$ & $\begin{array}{c}P_{1}=0.002^{*} \\
P_{2} \& P_{3}=0.0001^{*}\end{array}$ \\
\hline HOMA-IR index & $\begin{array}{l}0.19-1.52 \\
0.79 \pm 0.37\end{array}$ & $\begin{array}{l}1.09-3.89 \\
2.24 \pm 0.88\end{array}$ & $\begin{array}{l}2.18-11.37 \\
5.72 \pm 2.18\end{array}$ & $\begin{array}{l}67.876 \\
0.0001^{*}\end{array}$ & $\begin{array}{c}P_{1}=0.006^{*} \\
P_{2} \& P_{3}=0.0001^{*}\end{array}$ \\
\hline $\mathrm{TC}(\mathrm{mg} / \mathrm{ml})$ & $\begin{array}{c}99.00-210.00 \\
159.55 \pm 35.40\end{array}$ & $\begin{array}{l}173.00-250.00 \\
208.00 \pm 24.75\end{array}$ & $\begin{array}{l}209.00-290.00 \\
245.90 \pm 23.91\end{array}$ & $\begin{array}{l}46.109 \\
0.0001^{*}\end{array}$ & $\begin{array}{c}P_{1} \& P_{2}=0.0001^{*} \\
P_{3}=0.0001^{*}\end{array}$ \\
\hline TG (mg/dl) & $\begin{array}{c}54.00-195.00 \\
117.45 \pm 42.44\end{array}$ & $\begin{array}{c}126.00-230.00 \\
176.7 \pm 27.99\end{array}$ & $\begin{array}{c}143.00-295.00 \\
219.8 \pm 39.18\end{array}$ & $\begin{array}{l}38.416 \\
0.0001^{*}\end{array}$ & $\begin{array}{c}P_{1}=0.002^{*} \\
P_{2}=0.0001^{*} \\
P_{3}=0.002^{*}\end{array}$ \\
\hline HDL-C (mg/dl) & $\begin{array}{c}22.00-77.00 \\
48.05 \pm 14.52\end{array}$ & $\begin{array}{c}30.00-88.00 \\
63.15 \pm 15.42\end{array}$ & $\begin{array}{c}22.00-74.00 \\
42.50 \pm 12.46\end{array}$ & $\begin{array}{l}11.344 \\
0.0001^{*}\end{array}$ & $\begin{array}{l}P_{1}=0.006^{*} \\
P_{2}=0.470 \\
P_{3}=0.0001^{*}\end{array}$ \\
\hline LDL-C (mg/dl) & $\begin{array}{l}28.40-155.00 \\
88.01 \pm 37.04\end{array}$ & $\begin{array}{c}50.40-178.00 \\
109.52 \pm 33.63\end{array}$ & $\begin{array}{l}94.50-206.00 \\
156.6 \pm 32.11\end{array}$ & $\begin{array}{l}20.913 \\
0.0001^{*}\end{array}$ & $\begin{array}{c}P_{1}=0.150 \\
P_{2} \& P_{3}=0.0001^{*}\end{array}$ \\
\hline VLDL (mg/dl) & $\begin{array}{l}10.80-39.00 \\
23.49 \pm 8.49\end{array}$ & $\begin{array}{l}25.20-46.00 \\
35.33 \pm 5.60\end{array}$ & $\begin{array}{c}28.60-94.50 \\
47.63 \pm 13.18\end{array}$ & $\begin{array}{l}31.544 \\
0.0001^{*}\end{array}$ & $\begin{array}{c}P_{1} \& P_{3}=0.001^{*} \\
P_{2}=0.0001^{*}\end{array}$ \\
\hline
\end{tabular}

Data are presented as range, mean \pm standard deviation. FPG: Fasting plasma glucose; FPI: Fasting plasma insulin; HOMA-IR: homeostasis model assessment for insulin resistance; TC: total cholesterol; TG: triglycerides; HDL-C: high density lipoprotein cholesterol; VLDL: very low density lipoprotein; LDL-C: low density lipoprotein cholesterol. G1: non-pregnant group; G2: normal pregnant group; G3: preeclamptic group. $P_{1}$ indicates significance difference between non-pregnant and normal pregnant group (G1 vs G2); $P_{2}$ indicates significance difference between non-pregnant and preeclamptic group (G1 vs G3); $P_{3}$ indicates significance difference between normal pregnant and preeclamptic group (G2 vs G3). * Significant difference $(p<0.05)$. 
Table 4. Castelli indices, oxidative stress marker, adipokines, inflammatory and endothelial dysfunction markers among the three study groups at enrollment $(n=60)$

\begin{tabular}{|c|c|c|c|c|c|}
\hline $\begin{array}{l}\text { Laboratory } \\
\text { findings }\end{array}$ & $\begin{array}{l}\text { Non-pregnant women } \\
\quad(\mathrm{G} 1 ; n=20)\end{array}$ & $\begin{array}{c}\text { Normally pregnant } \\
\text { women }(G 2 ; n=20)\end{array}$ & $\begin{array}{l}\text { Pregnant women with } \\
\text { preeclampsia }(G 3 ; n=20)\end{array}$ & $\begin{array}{c}\text { F value } \\
P\end{array}$ & Scheffe test $(P)$ \\
\hline $\begin{array}{l}\text { Castelli index-I } \\
\text { (TC/HDL-C) }\end{array}$ & $\begin{array}{c}1.72-7.14 \\
3.67 \pm 1.56\end{array}$ & $\begin{array}{c}1.96-6.57 \\
3.56 \pm 1.25\end{array}$ & $\begin{array}{l}3.34-10.73 \\
6.23 \pm 1.86\end{array}$ & $\begin{array}{l}18.354 \\
0.0001^{*}\end{array}$ & $\begin{array}{c}P_{1}=0.976 \\
P_{2} \& P_{3}=0.0001^{*}\end{array}$ \\
\hline $\begin{array}{l}\text { Castelli index-II } \\
\text { (LDL/HDL-C) }\end{array}$ & $\begin{array}{c}0.41-5.65 \\
2.15 \pm 1.46\end{array}$ & $\begin{array}{c}0.57-4.68 \\
1.95 \pm 1.06\end{array}$ & $\begin{array}{c}1.49-7.81 \\
4.04 \pm 1.62\end{array}$ & $\begin{array}{c}13.655 \\
0.0001^{*}\end{array}$ & $\begin{array}{c}P_{1}=0.904 \\
P_{2} \& P_{3}=0.0001^{*}\end{array}$ \\
\hline $\mathrm{MDA}(\mu \mathrm{mole} / \mathrm{ml})$ & $\begin{array}{l}1.67-4.62 \\
2.97 \pm 0.93\end{array}$ & $\begin{array}{c}1.89-5.87 \\
3.62 \pm 1.07\end{array}$ & $\begin{array}{c}2.26-6.75 \\
4.65 \pm 1.41\end{array}$ & $\begin{array}{l}10.712 \\
0.0001^{*}\end{array}$ & $\begin{array}{c}P_{1}=0.217 \\
P_{2}=0.0001^{*} \\
P_{3}=0.024^{*}\end{array}$ \\
\hline Resistin (ng/ml) & $\begin{array}{c}3.88-7.75 \\
5.78 \pm 1.04\end{array}$ & $\begin{array}{l}3.55-11.95 \\
6.48 \pm 2.00\end{array}$ & $\begin{array}{l}4.77-15.66 \\
8.80 \pm 2.47\end{array}$ & $\begin{array}{c}13.503 \\
0.0001^{*}\end{array}$ & $\begin{array}{c}P_{1}=0.520 \\
P_{2}=0.0001^{*} \\
P_{3}=0.002^{*}\end{array}$ \\
\hline Vaspin (ng/ml) & $\begin{array}{c}9.05-17.77 \\
13.69 \pm 2.43\end{array}$ & $\begin{array}{l}10.08-18.55 \\
14.57 \pm 2.16\end{array}$ & $\begin{array}{c}9.88-22.53 \\
17.18 \pm 3.38\end{array}$ & $\begin{array}{c}8.946 \\
0.0001^{*}\end{array}$ & $\begin{array}{l}P_{1}=0.588 \\
P_{2}=0.001^{*} \\
P_{3}=0.014^{*}\end{array}$ \\
\hline IL-18 (pg/ml) & $\begin{array}{l}68.32-122.46 \\
92.36 \pm 13.53\end{array}$ & $\begin{array}{l}83.10-127.54 \\
102.62 \pm 12.0\end{array}$ & $\begin{array}{l}83.10-136.22 \\
113.16 \pm 15.1\end{array}$ & $\begin{array}{l}11.676 \\
0.0001^{*}\end{array}$ & $\begin{array}{c}P_{1}=0.067 \\
P_{2} \& P_{3}=0.0001^{*}\end{array}$ \\
\hline ADMA $(\mu \mathrm{mole} / \mathrm{l})$ & $\begin{array}{c}0.24-1.72 \\
0.55 \pm 0.34\end{array}$ & $\begin{array}{c}0.26-1.06 \\
0.59 \pm 0.50\end{array}$ & $\begin{array}{c}0.12-1.03 \\
0.66 \pm 0.28\end{array}$ & $\begin{array}{l}0.610 \\
0.547\end{array}$ & $\begin{array}{l}P_{1}=0.919 \\
P_{2}=0.553 \\
P_{3}=0.793\end{array}$ \\
\hline
\end{tabular}

Data are presented as range, mean \pm standard deviation. ADMA: asymmetric dimethyl arginine; MDA: malondialdehyde, IL-18: interleukin-18. G1: non-pregnant group; G2: normal pregnant group; G3: preeclamptic group. $P_{1}$ indicates significance difference between non-pregnant and normal pregnant group (G1 vs G2); $P_{2}$ indicates significance difference between non-pregnant and preeclamptic group (G1 vs G3); $P_{3}$ indicates significance difference between normal pregnant and preeclamptic group (G2 vs G3). * Significant difference $(p<0.05)$.

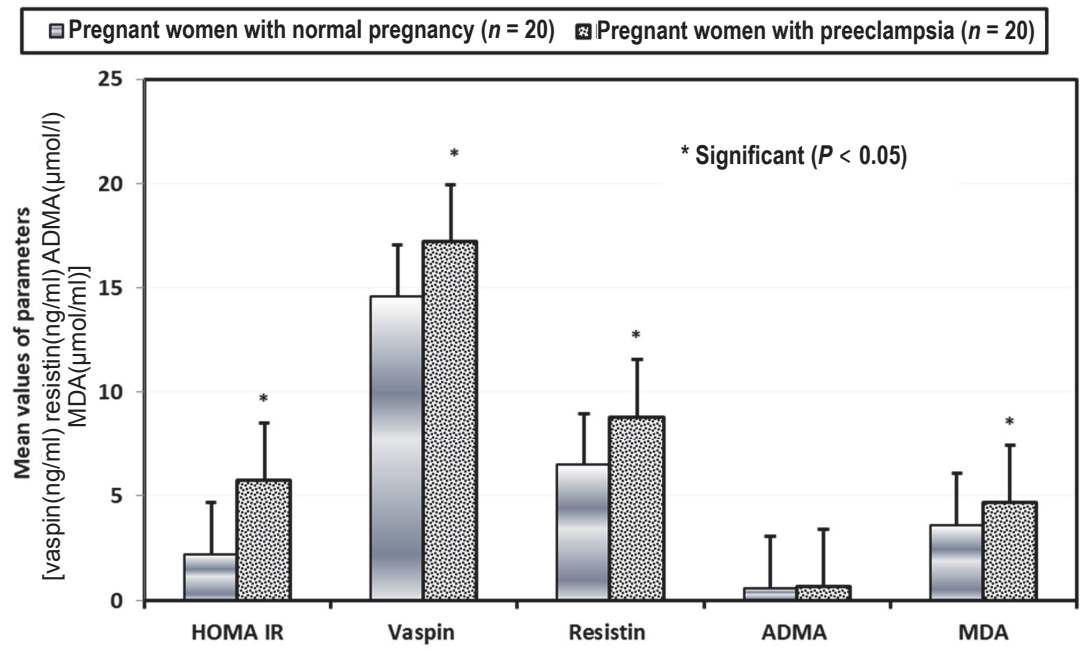

Fig. 1. Mean values of HOMA-IR, vaspin, resistin, ADMA and MDA among the pregnant women with and without preeclampsia during their third trimester of pregnancy

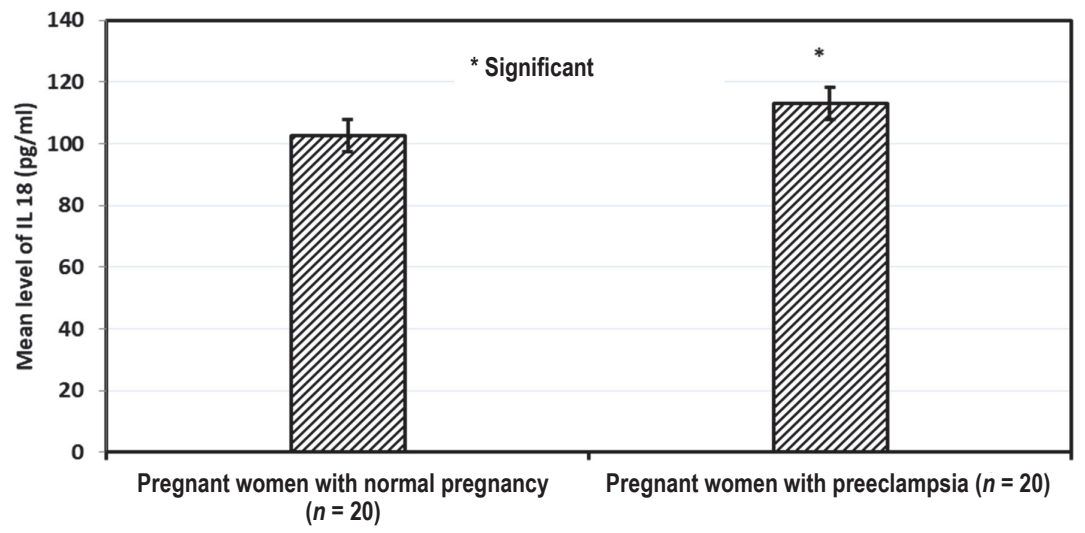

Fig. 2. Mean values of IL-18 among the pregnant women with and without preeclampsia during their third trimester of pregnancy 
Serum resistin and vaspin levels were significantly elevated in preeclamptic women than non-pregnant and normally pregnant women $(P<0.05)$. However, the levels of these adipokines were non-significantly elevated in normally pregnant women as compared to non-pregnant women $\left(P_{1}=0.52, P_{1}=0.58 \mathrm{re}-\right.$ spectively). Serum MDA and serum IL-18 concentrations were significantly elevated during preeclampsia as compared to the other two groups $(P<0.05)$. The levels of the oxidative stress and inflammatory markers were non-significantly elevated in normally pregnant women as compared to the control group $(P>0.05)$. Serum ADMA concentration showed non-significant difference among all study groups $\left(P_{1}=0.919 ; P_{2}=0.55\right.$; $P_{3}=0.79$ respectively).

Tables 5 and 6 illustrate the laboratory findings in pregnant women with and without preeclampsia during the third trimester of pregnancy and four weeks after delivery. Four weeks after delivery, the preeclamptic women showed significantly lower fasting blood glucose, insulin level and HOMA-IR index as compared to their third trimester values ( $P=0.004, P=0.0001, P=0.0001$ respectively). As regarding women with normal pregnancy, however blood glucose was non-significantly decreased postpartum $(P=0.897)$, insulin level and HOMA-IR were significantly decreased four weeks after delivery as compared to their third trimester values
$(P=0.001)$. In preeclamptic group, TC, TG, LDL- $C$ levels showed non-significant decrease four weeks after delivery as compared to their antepartum levels $(p>0.05)$. In contrast, TC and TG levels showed significant decrease in normally pregnant women after delivery $(P=0.0001)$. Also, VLDL concentration was significantly decreased in pregnant women with or without preeclampsia four weeks after delivery $(p<0.05)$. One month after delivery, Castelli risk indices I and II showed non-significant decrease in preeclamptic group as compared to their third trimester values $(P>0.05)$. As compared to the third trimester data, the preeclamptic women showed non-significant increase in HDL-C concentrations $(P=0.688)$ while normally pregnant women showed significant decrease in HDL- $C$ level $(P=0.0001)$ four weeks after delivery. Serum MDA, IL-18 and ADMA levels were non-significantly changed postpartum in both preeclamptic and normally pregnant women $(P>0.05)$. Although, serum resistin and vaspin levels were significantly decreased four weeks postpartum in preeclamptic group as compared to their third trimester values $(P<0.05)$, women with normal pregnancy showed non-significant decrease in the levels of these adipokines after delivery $(P>0.05)$. Mean values of serum resistin, vaspin, ADMA and MDA among the pregnant women with and without preeclampsia are shown in Fig. 3.

Table 5. Glycemic and lipid panels among pregnant women with and without preeclampsia during their third trimester and four weeks after delivery $(n=40)$

\begin{tabular}{|c|c|c|c|c|c|c|}
\hline \multirow[t]{2}{*}{ Laboratory findings } & \multicolumn{2}{|c|}{$\begin{array}{l}\text { Normally pregnant women } \\
(\mathrm{G} 2 ; n=20)\end{array}$} & \multicolumn{2}{|c|}{$\begin{array}{l}\text { Preeclamptic women } \\
\quad(G 3 ; n=20)\end{array}$} & \multirow[t]{2}{*}{$t$-test } & \multirow[t]{2}{*}{$P$} \\
\hline & Mean \pm SD & $\begin{array}{c}\text { Paired } t \text {-test } \\
P \text {-value }\end{array}$ & Mean \pm SD & $\begin{array}{c}\text { Paired } t \text {-test } \\
P \text {-value }\end{array}$ & & \\
\hline \multicolumn{7}{|l|}{ FPG (mg/dl) } \\
\hline At 3rd trimester & $88.50 \pm 11.51$ & 0.130 & $112.8 \pm 15.94$ & 3.052 & 5.527 & $0.0001^{*}$ \\
\hline 4 Weeks postpartum & $89.00 \pm 12.82$ & 0.897 & $84.00-118.00$ & $0.004^{*}$ & 3.039 & $0.004^{*}$ \\
\hline \multicolumn{7}{|l|}{ FPI $(\mu \mathrm{IU} / \mathrm{ml})$} \\
\hline At 3rd trimester & $10.22 \pm 3.75$ & 3.735 & $20.18 \pm 6.89$ & 5.468 & 5.676 & $0.0001^{*}$ \\
\hline 4 Weeks postpartum & $6.44 \pm 2.53$ & $0.001^{*}$ & $10.90 \pm 3.17$ & $0.0001^{*}$ & 4.924 & $0.0001^{*}$ \\
\hline \multicolumn{7}{|l|}{ HOMA-IR index } \\
\hline At 3rd trimester & $2.24 \pm 0.88$ & 3.448 & $5.72 \pm 2.18$ & 5.858 & 6.615 & $0.0001^{*}$ \\
\hline 4 Weeks postpartum & $1.42 \pm 0.60$ & $0.001^{*}$ & $2.68 \pm 0.79$ & $0.0001^{*}$ & 5.714 & $0.0001^{*}$ \\
\hline \multicolumn{7}{|l|}{$\mathrm{TC}(\mathrm{mg} / \mathrm{dl})$} \\
\hline At 3rd trimester & $208.0 \pm 24.75$ & 4.536 & $245.9 \pm 23.91$ & 1.288 & 4.925 & $0.0001^{*}$ \\
\hline 4 Weeks postpartum & $175.9 \pm 19.72$ & $0.0001^{*}$ & $235.95 \pm 24.9$ & 0.205 & 8.448 & $0.0001^{*}$ \\
\hline \multicolumn{7}{|l|}{$\mathrm{TG}(\mathrm{mg} / \mathrm{dl})$} \\
\hline At 3rd trimester & $176.7 \pm 27.99$ & 6.506 & $219.8 \pm 39.18$ & 1.848 & 4.003 & $0.0001^{*}$ \\
\hline 4 Weeks postpartum & $122.2 \pm 24.85$ & $0.0001^{*}$ & $200.3 \pm 26.07$ & 0.072 & 9.699 & $0.0001^{*}$ \\
\hline \multicolumn{7}{|l|}{$\mathrm{HDL}-\mathrm{C}(\mathrm{mg} / \mathrm{dl})$} \\
\hline At 3rd trimester & $63.15 \pm 15.42$ & 4. 680 & $42.50 \pm 12.46$ & 0.405 & 4.658 & $0.0001^{*}$ \\
\hline 4 Weeks postpartum & $43.30 \pm 11.04$ & $0.0001^{*}$ & $44.00 \pm 10.92$ & 0.688 & 0.202 & 0.841 \\
\hline \multicolumn{7}{|l|}{$\mathrm{LDL}-\mathrm{C}(\mathrm{mg} / \mathrm{dl})$} \\
\hline At 3rd trimester & $109.5 \pm 33.63$ & 0.152 & $156.6 \pm 32.11$ & 0.442 & 4.531 & $0.0001^{*}$ \\
\hline 4 Weeks postpartum & $108.2 \pm 21.73$ & 0.880 & $152.4 \pm 28.59$ & 0.661 & 5.508 & $0.0001^{*}$ \\
\hline \multicolumn{7}{|l|}{ VLDL (mg/dl) } \\
\hline At 3rd trimester & $35.33 \pm 5.60$ & 6.506 & $47.63 \pm 13.18$ & 2.555 & 3.842 & $0.0001^{*}$ \\
\hline 4 Weeks postpartum & $24.44 \pm 4.97$ & $0.0001^{*}$ & $39.56 \pm 5.09$ & $0.015^{*}$ & 9.504 & $0.0001^{*}$ \\
\hline
\end{tabular}

Data are presented as mean \pm standard deviation. FPG: Fasting plasma glucose; FPI: Fasting plasma insulin; HOMA-IR: homeostasis model assessment for insulin resistance; TC: total cholesterol; TG: triglycerides; HDL-C: high density lipoprotein cholesterol; VLDL: very low density lipoprotein; LDL-C: low density lipoprotein cholesterol. * Significant difference $(p<0.05)$. 
Table 6. Castelli indices, oxidative stress marker, adipokines, inflammatory and endothelial dysfunction markers among pregnant women with and without preeclampsia during their third trimester and four weeks after delivery $(n=40)$

\begin{tabular}{|c|c|c|c|c|c|c|}
\hline \multirow[t]{2}{*}{ Laboratory findings } & \multicolumn{2}{|c|}{$\begin{array}{l}\text { Normally pregnant women } \\
\qquad(\mathrm{G} 2 ; n=20)\end{array}$} & \multicolumn{2}{|c|}{$\begin{array}{l}\text { Preeclamptic women } \\
\qquad(\mathrm{G} 3 ; n=20)\end{array}$} & \multirow[t]{2}{*}{$t$-test } & \multirow[t]{2}{*}{$P$} \\
\hline & Mean \pm SD & $\begin{array}{c}\text { Paired } t \text {-test } \\
P \text {-value }\end{array}$ & Mean \pm SD & $\begin{array}{c}\text { Paired } t \text {-test } \\
P \text {-value }\end{array}$ & & \\
\hline \multicolumn{7}{|c|}{ Castelli index-I (TC/HDL-C) } \\
\hline At 3rd trimester & $3.56 \pm 1.25$ & 1.827 & $6.23 \pm 1.86$ & 1.035 & 5.328 & $0.0001^{*}$ \\
\hline 4 Weeks postpartum & $4.31 \pm 1.36$ & 0.076 & $5.67 \pm 1.56$ & 0.307 & 2.925 & $0.006^{*}$ \\
\hline \multicolumn{7}{|c|}{ Castelli index-II (LDL/HDL-C) } \\
\hline At 3rd trimester & $1.95 \pm 1.06$ & 2.111 & $4.04 \pm 1.62$ & 0.689 & 4.842 & $0.0001^{*}$ \\
\hline 4 Weeks postpartum & $2.72 \pm 1.24$ & $0.042^{*}$ & $3.72 \pm 1.36$ & 0.495 & 2.430 & $0.020^{*}$ \\
\hline \multicolumn{7}{|l|}{ MDA ( $\mu$ mole/ml) } \\
\hline At 3rd trimester & $3.62 \pm 1.07$ & 1.647 & $4.65 \pm 1.41$ & 1.573 & 2.596 & $0.013^{*}$ \\
\hline 4 Weeks postpartum & $3.10 \pm 0.92$ & 0.108 & $4.06 \pm 0.91$ & 0.124 & 3.304 & $0.002^{*}$ \\
\hline \multicolumn{7}{|l|}{ Resistin (ng/ml) } \\
\hline At 3rd trimester & $6.48 \pm 2.00$ & 1.730 & $8.80 \pm 2.47$ & 2.728 & 3.276 & $0.002^{*}$ \\
\hline 4 Weeks postpartum & $5.60 \pm 1.07$ & 0.092 & $7.06 \pm 1.43$ & $0.010^{*}$ & 3.667 & $0.001^{*}$ \\
\hline \multicolumn{7}{|l|}{ Vaspin $(\mathrm{ng} / \mathrm{ml})$} \\
\hline At 3rd trimester & $14.57 \pm 2.16$ & 1.908 & $17.18 \pm 3.38$ & 3.156 & 2.898 & $0.006^{*}$ \\
\hline 4 Weeks postpartum & $13.33 \pm 1.96$ & 0.064 & $14.44 \pm 1.88$ & $0.003^{*}$ & 1.835 & 0.074 \\
\hline \multicolumn{7}{|l|}{ IL-18 (pg/ml) } \\
\hline At 3rd trimester & $102.62 \pm 12.0$ & 0.663 & $113.16 \pm 15.1$ & 0.777 & 2.441 & $0.019^{*}$ \\
\hline 4 Weeks postpartum & $100.57 \pm 6.81$ & 0.511 & $110.14 \pm 8.61$ & 0.442 & 3.900 & $0.0001^{*}$ \\
\hline \multicolumn{7}{|l|}{ ADMA ( $\mu$ mole/l) } \\
\hline At 3rd trimester & $0.59 \pm 0.50$ & 0.293 & $0.66 \pm 0.28$ & 0.133 & 0.736 & 0.466 \\
\hline 4 Weeks postpartum & $0.57 \pm 0.34$ & 0.771 & $0.67 \pm 0.33$ & 0.895 & 0.992 & 0.327 \\
\hline
\end{tabular}

Data are presented as mean \pm standard deviation. ADMA: asymmetric dimethyl arginine; MDA: malondialdehyde; IL-18: interleukin-18. * Significant difference $(p<0.05)$.

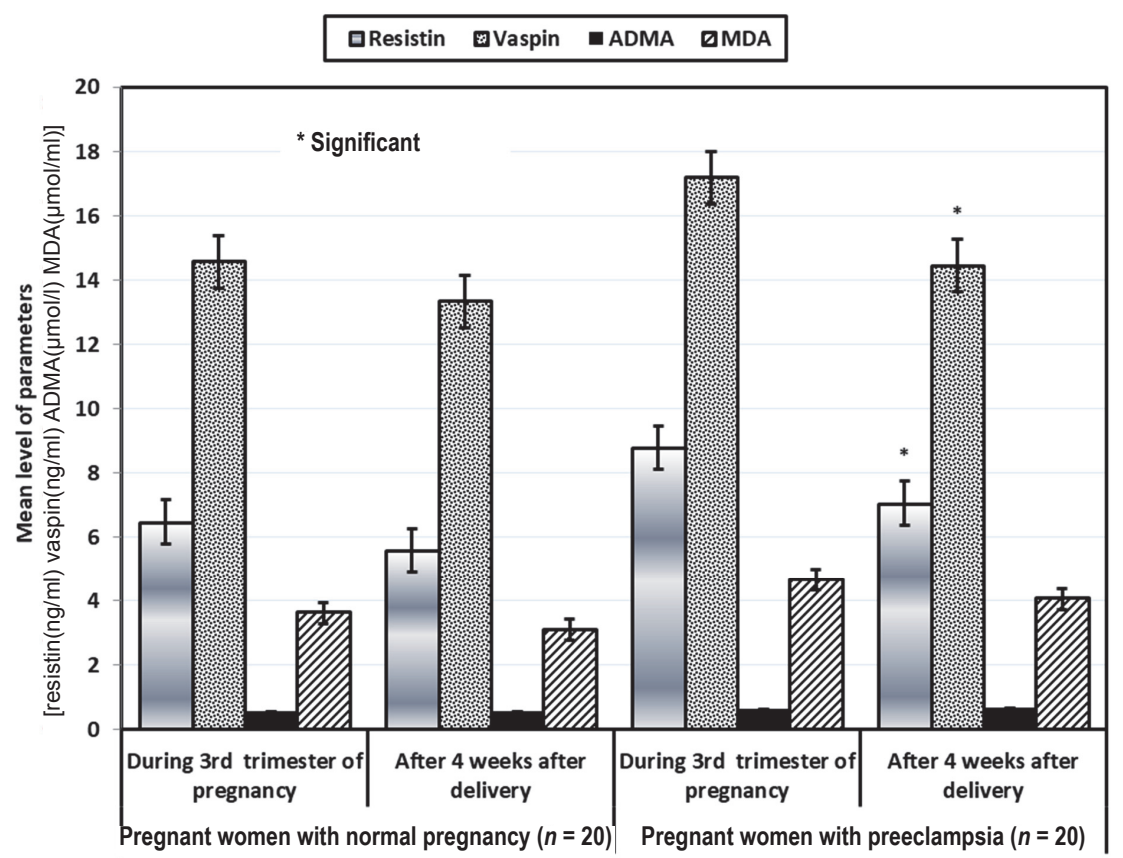

Fig. 3. Mean values of resistin, vaspin, ADMA and MDA among the pregnant women with and without preeclampsia during their third trimester of pregnancy and 4 weeks after delivery 


\section{Discussion}

Preeclampsia is a pregnancy related complication that can impair both mothers and babies health. Our study illustrates that, all the pregnant women with preeclampsia showed $\mathrm{SBP} \geq 140 \mathrm{mmHg}$ and DBP $\geq 90 \mathrm{mmHg}$, a result seems parallel to previously reported findings (Peres et al., 2018).

Spot urine protein : creatinine ratio was used as an alternative to 24-hour urine protein measurement for the quantitative detection of proteinuria since spot urine protein : creatinine ratio was reported to be goodly correlated with 24 hours proteinuria (Demirci et al., 2015). During the current study, all women with preeclampsia exhibited protienuria on urine dipstick test and showed significantly higher urinary $\mathrm{P} / \mathrm{C}$ ratio as compared to non-pregnant and normally pregnant women, a result seems in accordance with previously reported findings (Demirci et al., 2015).

Our study revealed that, ante-partum fasting blood glucose level, insulin level and HOMA-IR (the favorable index for insulin resistance) were significantly elevated in preeclamptic women as compared to non-pregnant and normally pregnant women; a result comes in matching with previously reported findings (Girouard et al., 2007). Four weeks after delivery, HOMA-IR index showed significant decrease in pregnant women with or without preeclampsia. The improvement of insulin resistance after delivery is attributed to the removal of the placenta which releases certain hormones that may counteract insulin action (Ryan and Enns, 1988). In this context, insulin resistance may be involved in the pathogenesis of preeclampsia and may represent one of the risk factors that can help in the prediction of preeclampsia.

During the third trimester of pregnancy, HDL-C level was significantly lower while TC, TG, LDL-C and VLDL levels were significantly higher in preeclamptic group than non-pregnant and normally pregnant groups. Our finding concerning HDL-C is in matching with formerly reported findings (Islam et al., 2010). The observed elevation of LDL-C level in preeclamptic women may be attributed to elevated estrogen and progesterone levels during preeclampsia (Salameh and Mastrogiannis, 1994). The observed higher VLDL level in preeclamptic women may be attributed to hypertriglyceridemia since VLDL is well known to transport TG in peripheral blood. Our result concerning VLDL comes in consonance with formerly reported findings (Herrera et al., 1988). The observed atherogenic lipid profile in preeclamptic women during the current study confirms the notion that, the impairment of the lipoprotein metabolism may play a critical role in the pathogenesis of preeclampsia and in the development of insulin resistance (Bassi et al., 2011). Hypertriglyceridemia with subsequent triglycerides accumulation can result in lipotoxicity and oxidative stress with subsequent impairment of glucose-stimulated insulin secretion and insulin resistance (Bassi et al., 2011). Castelli risk index I (CRI-I = TC/HDL) and Castelli risk index II $(\mathrm{CRI}-\mathrm{II}=\mathrm{LDL} / \mathrm{HDL})$ are more accurate risk predictors of coronary artery disease (CAD) than individual lipid parameters (Kannel, 2005). During the current study, preeclamptic women showed significantly higher CRI-I and CRI-II than normally pregnant and non-pregnant women suggesting their susceptibility for CAD and cardiovascular complications. Our result seems in accordance with previously reported findings revealed that; $\mathrm{TC} / \mathrm{HDL}$ ratio was significantly increased in women with preeclampsia as compared to normally pregnant women (Singh et al., 2015).
Preeclampsia is associated with increased oxidative stress and reduced placental antioxidant enzyme activity with subsequent endothelial dysfunction and oxidative injury in both maternal and placental compartment (Bassi et al., 2011). The present data revealed that, MDA level was significantly higher in preeclamptic women than non-pregnant and normally pregnant women. Our result is in parallel with a former study postulated that, MDA level was elevated during preeclampsia as compared to normal pregnancy secondary to abnormally increased lipid peroxides formation by placenta during preeclampsia (Fenzl et al., 2013). During the current study, serum MDA concentrations tended to be decreased in both pregnancy groups after delivery. Our findings are in matching with aforementioned data postulated that, pregnancy is a stressful condition associated with accumulation of oxidative stress markers which may play a considerable role in the development of insulin resistance (Bassi et al., 2011).

Resistin is an adipokine secreted by macrophages, monocytes and white adipose tissue. Resistin promotes inflammation and contributes to insulin resistance through AMP-activated protein kinase dependent and independent mechanisms (Roca-Rodríguez et al., 2012; Yura et al., 2003). Our data postulated that, circulating resistin level was significantly higher in preeclamptic group than the other two groups and its level showed significant decline four weeks after delivery. Our former result comes in accordance with previously reported findings (Seol et al., 2010). These aforementioned data suggest that, the elevation of resistin level during preeclampsia is not only related to increased secretions from placenta and adipose tissue but also may be attributed to other sources responsible for increased circulating resistin level. Monocytes which are regulated by many inflammatory cytokines may represent another source for circulating resistin (Kaser et al., 2003). Preeclampsia is a systemic inflammatory disease associated with monocytes activation, therefore the observed elevation in resistin level during preeclampsia may be attributed to the systemic inflammatory response (Filková et al., 2009). Vaspin is an adipokine which sensitizes insulin and regulates endogenous glucose metabolism (Hida et al., 2005). We observed that, circulating vaspin was significantly elevated in preeclamptic women than normally pregnant and non-pregnant women and its level showed significant decline four weeks after delivery in women with preeclampsia. The significant elevation of serum vaspin concentration in preeclamptic women during their third trimester may be attributed to a compensatory protective mechanism against insulin resistance and inflammation through the anti-inflammatory activity of vaspin (Hida et al., 2005). In contradiction with our finding, Stepan et al. (2010) reported that, there was non-significant difference in circulating vaspin levels between preeclamptic women and women in the control group (Stepan et al., 2010).

Interleukin-18 originally described as interferon gamma (IFN-c) inducing factor is a pro-inflammatory cytokine which may be associated with both inflammation and insulin resistance. IFN-c could inhibit the migration and invasion of cytotrophoblasts and triggers spiral artery modification which may be related to the etiology of the preeclampsia (Monk et al., 2005). Serum IL-18 concentration was significantly elevated during preeclampsia than non-pregnant and normally pregnant women. This elevation of IL-18 in women with preeclamsia may be attributed to preeclampsia associated systemic inflammatory response. The result obtained with IL-18 during the current study is in matching with other authors who reported higher IL-18 level during preeclampsia as compared to 
normal pregnancy (Seol et al., 2009). In contrast, our result seems in disagreement with a previously reported finding reported lower IL-18 level in preeclamptic women as compared to normotensive pregnant controls (Laskowska et al., 2011).

The endogenous inhibitor of nitric oxide synthesis, asymmetric dimethyl arginine (ADMA) may represent an important factor for endothelial dysfunction during pregnancy (Slaghekke et al., 2006). ADMA concentrations showed non-significant difference among all study groups. In addition, there was non-significant difference between post-partum and third trimester ADMA concentrations for both pregnant groups. Our former result may be attributed to a state of possible vasodilation that may occur during pregnancy. Our finding comes in matching with a previously reported finding reported absence of significant difference in circulating ADMA concentration among women with preeclampsia, normally pregnant women and non-pregnant women (Silver et al., 1996). However, our result seems in contradiction with other former finding demonstrated abnormal decline in ADMA concentration during pregnancy (Begum et al., 1996).

During the current study, preeclamptic women showed earlier delivery with low birth weight neonates and the majority of them underwent cesarean section for delivery. These consequences may be attributed to preeclampsia associated insulin resistance, dyslipidemia, oxidative stress and inflammation. Therefore, practitioners should consider regular women's prenatal clinic visits for monitoring of their blood pressures, asking for regular screening of proteinuria, evaluation of blood glucose, assessment of sFlt-1/ PlGF ratio and evaluation of lipid panel with management of hyperlipidemia. Although, there are discrepancies about their teratogenicity (Edison and Muenke, 2004; Winterfeld et al., 2013), statin derivatives are still used during preeclampsia for their antihyperlipidemic effect and their lipid independent pleiotropic effects. Statin derivatives diminish inflammation and oxidative stress, inhibit the coagulation cascade and protect the endothelium (Girardi, 2014). Statins may increase the expression of Heme Oxygenase-1 (Hmox-1), the enzyme with anti-inflammatory and antioxidant properties. Stimulated Hmox-1 inhibits the antiangiogenic factors soluble fms-like tyrosine kinase-1 (sFlt-1) and soluble endoglin (sEng) and directly improves endothelial dysfunction (Brownfoot et al., 2015). To minimize the negative outcome, pravastatin the more hydrophilic statin derivative unable to pass through the placenta may represent a suitable candidate to be used for preeclampsia (Ahmed et al., 2020; Winterfeld et al., 2013).

Finally, it is worth mentioning that, the sample size used during the current study was based on some previous studies conducted on preeclampsia (Speer et al., 2008). Furthermore, the points of strength of our study include its cohort prospective design and the assessment of many biological markers which may be involved in the pathogenesis of preeclampsia.

\section{Conclusions}

The data obtained during the current study revealed that, preeclampsia was associated with insulin resistance, dyslipidemia, oxidative stress and inflammation suggesting their role in the pathogenesis of preeclampsia. Furthermore, the significant elevation of serum resistin and vaspin levels during the third trimester of pregnancy and their significant decline four weeks after delivery in preeclamptic women focus the attention on the role played by these adipokines in the pathogenesis of preeclampsia. However, large scale studies are still required.

\section{Study limitation}

The relatively small sample size represents the main limitation of the current study.

\section{Conflict of interests}

The authors declare that, there is no conflict of interests.

\section{Acknowledgement}

We are so thankful to our participants. We offer our appreciation to Dr. Emad Ali El-Saadawy for his help.

\section{Funding}

This research did not receive any specific grant from funding agencies.

\section{Author contributions}

Tarek Mohamed Mostafa, reviewed the literature, constructed the study design. Assessment and enrolment of participants was carried out by Haitham Aboali Hamza. Amany Yasseen Talab collected the clinical data and required samples. Amany Yasseen Talab and Tarek Mohamed Mostafa performed laboratory investigation of collected samples. All authors shared in performing statistical analysis and writing the manuscript. All authors approved the final manuscript.

\section{References}

Abhari FR, Ghanbari Andarieh M, Farokhfar A, Ahmady S (2014). Estimating rate of insulin resistance in patients with preeclampsia using HOMA-IR index and comparison with nonpreeclampsia pregnant women. Biomed Res Int 2014: 140851. DOI: $10.1155 / 2014 / 140851$.

Ahmed A, Williams DJ, Cheed V, Middleton LJ, Ahmad S, Wang K, et al. (2020). Pravastatin for early-onset preeclampsia: a randomized, blinded, placebo-controlled trial. BJOG 127(4): 478-488. DOI: 10.1111/1471-0528.16013.

Bassi R, Kaur M, Sharma S (2011). Study of changes in lipid profile, lipid peroxidation and superoxide dismutase during normal pregnancy. Indian Journal of Fundamental and Applied Life Sciences 1(3): 249-254.

Begum S, Yamasaki M, Mochizuki M (1996). Urinary levels of nitric oxide metabolites in normal pregnancy and preeclampsia. J Obstet Gynaecol Res 22(6): 551-559. DOI: 10.1111/j.1447-0756.1996. tb01070.x

Brown MA, Lindheimer MD, de Swiet M, Van Assche A, Moutquin JM (2001). The classification and diagnosis of the hypertensive disorders of pregnancy: statement from the International Society for the Study of Hypertension in Pregnancy (ISSHP). Hypertens Pregnancy 20(1): IX-XIV. DOI: 10.1081/PRG100104165.

Brownfoot FC, Tong S, Hannan NJ, Binder NK, Walker SP, Cannon P, et al. (2015). Effects of pravastatin on human placenta, endothelium, and women with severe preeclampsia. Hypertension 66(3): 687-697. DOI: 10.1161/HYPERTENSIONAHA.115.05445.

Chen C, Jiang J, Lü JM, Chai H, Wang X, Lin PH, et al. (2010).

Resistin decreases expression of endothelial nitric oxide synthase through oxidative stress in human coronary artery endothelial cells. Am J Physiol Heart Circ Physiol 299(1): H193-H201. DOI: 10.1152/ajpheart.00431.2009.

de Lucca L, Rodrigues F, Jantsch LB, Kober H, Neme WS, Gallarreta FMP, et al. (2016). Delta-aminolevulinate dehydratase activity and oxidative stress markers in preeclampsia. Biomed Pharmacother 84: 224-229. DOI: 10.1016/j.biopha.2016.09.033.

Demirci O, Kumru P, Arınkan A, Ardıç C, Arısoy R, Tozkır E, et al. (2015). Spot protein/creatinine ratio in preeclampsia as an alternative for 24-hour urine protein. Balkan Med J 32(1): 51-55. DOI: 10.5152/balkanmedj.2015.15447. 
Draper HH, Hadley M (1990). Malondialdehyde determination as index of lipid Peroxidation. Methods Enzymol 186: 421-431. DOI: 10.1016/0076-6879(90)86135-i.

Edison RJ, Muenke M (2004). Central nervous system and limb anomalies in case reports of first-trimester statin exposure. N Engl J Med 350 (15): 1579-1582. DOI: 10.1056/ NEJM200404083501524.

Fenzl V, Flegar-Meštrić Z, Perkov S, Andrišić L, Tatzber F, Žarković N, et al. (2013). Trace elements and oxidative stress in hypertensive disorders of pregnancy. Arch Gynecol Obstet 287(1): 19-24. DOI: $10.1007 / \mathrm{s} 00404-012-2502-4$.

Filková M, Haluzík M, Gay S, Senolt L (2009). The role of resistin as a regulator of inflammation: Implications for various human pathologies. Clin Immunol 133(2): 157-170. DOI: 10.1016/j. clim.2009.07.013.

Friedewald WT, Levy RI, Fredrickson DS (1972). Estimation of the concentration of low density lipoprotein cholesterol in plasma without use of the preparative ultracentrifuge. Clin Chem 18(6): 499-502. DOI: 10.1093/clinchem/18.6.499.

Ghulmiyyah L, Sibai B (2012). Maternal mortality from preeclampsia/eclampsia. Semin Perinatol 36(1): 56-59. DOI: 10.1053/j.semperi.2011.09.011.

Girardi G (2014). Can statins prevent pregnancy complications? J Reprod Immunol 101-102: 161-167. DOI: 10.1016/j. jri.2013.07.005

Girouard J, Giguère Y, Moutquin JM, Forest JC (2007). Previous hypertensive disease of pregnancy is associated with alterations of markers of insulin resistance. Hypertension 49(5): 1056-1062. DOI: 10.1161/HYPERTENSIONAHA.107.087528.

Herrera E, Lasunción MA, Gomez-Coronado D, Aranda P, López-Luna P, Maier I (1988). Role of lipoprotein lipase activity on lipoprotein metabolism and the fate of circulating triglycerides in pregnancy. Am J Obstet Gynecol 158(6): 1575-1583. DOI: 10.1016/0002-9378(88)90193-7.

Hida K, Wada J, Eguchi J, Zhang H, Baba M, Seida A, et al. (2005). Visceral adipose tissue-derived serine protease inhibitor: a unique insulin-sensitizing adipocytokine in obesity. Proc Natl Acad Sci USA 102(30): 10610-10615.DOI: 10.1073/pnas.0504703102.

Hsu WY, Chao YW, Tsai YL, Lien CC, Chang CF, Deng MC, et al. (2011). Resistin induces monocyte-endothelial cell adhesion by increasing ICAM-1 and VCAM-1 expression in endothelial cells via p38MAPK-dependent pathway. J Cell Physiol 226: 2181-2188. DOI: $10.1002 /$ jcp. 22555 .

Islam N, Chowdhury M, Kibria G, Akhter S (2010). Study of serum lipid profile in pre-eclampsia and eclampsia. Faridpur Med Coll J 5(2): 56-59. DOI: 10.3329/fmcj.v5i2.6823.

Kannel WB (2005). Risk stratification of dyslipidemia: insights from the Framingham Study. Curr Med Chem Cardiovasc Hematol Agents 3(3): 187-193. DOI: 10.2174/1568016054368250.

Kaser S, Kaser A, Sandhofer A, Ebenbichler CF, Tilg H, Patsch JR (2003). Resistin messenger-RNA expression is increased by proinflammatory cytokines in vitro. Biochem Biophys Res Commun 309(2): 28--290. DOI: 10.1016/j.bbrc.2003.07.003.

Laskowska M, Laskowska K, Oleszczuk J (2011). Interleukin-18 concentrations in pregnancies complicated by preeclampsia with and without IUGR: A comparison with normotensive pregnant women with isolated IUGR and healthy pregnant women. Pregnancy Hypertens 1(3-4): 206-212. DOI: 10.1016/j. preghy.2011.07.003.

Lockwood CJ, Krikun G, Caze R, Rahman M, Buchwalder LF, Schatz F (2008). Decidual cell-expressed tissue factor in human pregnancy and its involvement in hemostasis and preeclampsia-related angiogenesis. Ann N Y Acad Sci 1127: 67-72. DOI: 10.1196/ annals.1434.013.

Matthews DR, Hosker JP, Rudenski AS, Naylor BA, Treacher DF, Turner RC (1985). Homeostasis model assessment: insulin resistance and $\beta$-cell function from fasting plasma glucose and insulin concentrations in man. Diabetologia 28(7): 412-419. DOI: $10.1007 / \mathrm{bf00280883.}$

Monk JM, Leonard S, McBey BA, Croy BA (2005). Induction of murine spiral artery modification by recombinant human interferon-gamma. Placenta 26(10): 835-838. DOI: 10.1016/j. placenta.2004.10.016.
Ohkuchi A, Hirashima C, Suzuki H, Takahashi K, Yoshida M, Matsubara S, et al. (2010). Evaluation of a new and automated electrochemiluminescence immunoassay for plasma sFlt-1 and PIGF levels in women with preeclampsia. Hypertens Res 33(5): 422-427. DOI: 10.1038/hr.2010.15.

Peres GM, Mariana M, Cairrão E (2018). Pre-eclampsia and eclampsia: An update on the pharmacological treatment applied in Portugal J Cardiovasc Dev Dis 5(1): 3. DOI: 10.3390/jcdd5010003.

Phalitakul S, Okada M, Hara Y, Yamawaki H (2011). Vaspin prevents TNF- $\alpha$-induced intracellular adhesion molecule- 1 via inhibiting reactive oxygen species-dependent $\mathrm{NF}-\kappa \mathrm{B}$ and $\mathrm{PKC} \theta$ activation in cultured rat vascular smooth muscle cells. Pharmacol Res 64 (5): 493-500. DOI: 10.1016/j.phrs.2011.06.001.

Roberts JM (1998). Endothelial dysfunction in preeclampsia. Semin Reprod Endocrinol 16(1): 5-15. DOI: 10.1055/s-2007-1016248.

Roca-Rodríguez MM, López-Tinoco C, Fernández-Deudero A, Murri M, García-Palacios MV, García-Valero MA, et al. (2012). Adipokines and metabolic syndrome risk factors in women with previous gestational diabetes mellitus. Diabetes Metab Res Rev 28(6): 542-548. DOI: 10.1002/dmrr.2313.

Ryan EA, Enns L (1988). Role of gestational hormones in the induction of IR. J Clin Endocrinol Metab 67(2): 341-347. DOI: 10.1210/jcem-67-2-341.

Salameh WA, Mastrogiannis DS (1994). Maternal hyperlipidemia in pregnancy. Clin Obstet Gynecol 37(1): 66-77. DOI: 10.1097/00003081-199403000-00011.

Seol HJ, Lee ES, Jung SE, Jeong NH, Lim JE, Park SH, et al. (2009). Serum levels of YKL-40 and interleukin-18 and their relationship to disease severity in patients with preeclampsia. J Reprod Immunol 79(2): 183-187. DOI: 10.1016/j.jri.2008.10.003.

Seol HJ, Oh MJ, Yeo MK, Kim A, Lee ES, Kim HJ (2010). Comparison of serum levels and the placental expression of resistin between patients with preeclampsia and normal pregnant women. Hypertens Pregnancy 29(3): 31--317. DOI: $10.3109 / 10641950902849850$.

Shah DA, Khalil RA (2015). Bioactive factors in uteroplacental and systemic circulation link placental ischemia to generalized vascular dysfunction in hypertensive pregnancy and preeclampsia. Biochem Pharmacol 95(4): 211-226. DOI: 10.1016/j.bcp.2015.04.012.

Silver RK, Kupferminc MJ, Russell TL, Adler L, Mullen TA, Caplan MS (1996). Evaluation of nitric oxide as a mediator of severe preeclampsia. Am J Obstet Gynecol 175(4): 1013-1017. DOI: 10.1016/s0002-9378(96)80044-5.

Singh M, Pathak MS, Paul A (2015). A Study on Atherogenic Indices of Pregnancy Induced Hypertension Patients as Compared to Normal Pregnant Women. J Clin Diagn Res 9(7): BC05-BC08. DOI: $10.7860 / J C D R / 2015 / 13505.6241$.

Slaghekke F, Dekker G, Jeffries B (2006). Endogenous inhibitors of nitric oxide and preeclampsia: a review. J Matern Fetal Neonatal Med 19(8): 447-452. DOI: 10.1080/14767050600852171.

Speer PD, Powers RW, Frank MP, Harger G, Markovic N, Roberts JM (2008). Elevated asymmetric dimethylarginine concentrations precede clinical preeclampsia, but not pregnancies with small-forgestational-age infants. Am J Obstet Gynecol 198(1): 112.e1-7. DOI: 10.1016/j.ajog.2007.05.052.

Stepan H, Kralisch S, Klostermann K, Schrey S, Reisenbüchler C, Verlohren M, et al. (2010). Preliminary report: circulating levels of the adipokine vaspin in gestational diabetes mellitus and preeclampsia. Metabolism 59(7): 1054-1056. DOI: 10.1016/j. metabol.2009.11.001.

Steppan CM, Bailey ST, Bhat S, Brown EJ, Banerjee RR, Wright CM, et al. (2001). The hormone resistin links obesity to diabetes. Nature 409(6818): 307-312. DOI: 10.1038/35053000.

Tousoulis D, Georgakis MK, Oikonomou E, Papageorgiou N, Zaromitidou M, Latsios G, et al. (2015). Asymmetric Dimethylarginine: Clinical Significance and Novel Therapeutic Approaches. Curr Med Chem 22(24): 2871-2901. DOI: 10.2174/0 929867322666150625095046

Tranquilli AL, Dekker G, Magee L, Roberts J, Sibai BM, Steyn W, et al. (2014). The classification, diagnosis and management of the hypertensive disorders of pregnancy: a revised statement from the ISSHP. Pregnancy Hypertens 4(2): 97-104. DOI: 10.1016/j. preghy.2014.02.001. 
Verma S, Li SH, Wang CH, Fedak PW, Li RK, Weisel RD, et al. (2003). Resistin promotes endothelial cell activation: further evidence of adipokine-endothelial interaction. Circulation 108(6): 736-740. DOI: 10.1161/01.CIR.0000084503.91330.49.

Winterfeld U, Allignol A, Panchaud A, Rothuizen LE, Merlob P, Cuppers-Maarschalkerweerd B, et al. (2013). Pregnancy outcome following maternal exposure to statins: a multicentre prospective study. BJOG 120 (4): 463-471. DOI: 10.1111/1471-0528.12066.

Yura S, Sagawa N, Itoh H, Kakui K, Nuamah MA, Korita D, et al. (2003). Resistin is expressed in the human placenta. J Clin Endocrinol Metab 88(3): 1394-1397. DOI: 10.1210/jc.2002011926. 